SELECCIONES MATEMÁTICAS
Universidad Nacional de Trujillo
ISSN: $2411-1783$ (Online)
2019; Vol.06(2): $196-203$.

\title{
Mathematical model for the study of the diffusion of Zika. Computational experimentation in Paramaribo and Santa Ana.
}

\section{Modelo Matemático para el estudio de la difusión del Zika. Experimentación computacional en Paramaribo y Santa Ana.}

\author{
Erick Manuel Delgado Moya *iD and Aymée Marrero Severo. ${ }^{\dagger}$
}

Received, Set. 16, 2019

Accepted, Nov. 15, 2019

How to cite this article:

Delegado, E., Marrero, A. Mathematical model for the study of the diffusion of Zika. Computational experimentation in Paramaribo and Santa Ana. Selecciones Matemáticas. 2019; 6(2):196-203. http://dx.doi.org/10.17268/sel. mat.2019.02.06

\begin{abstract}
Zika virus spreads to people primarily through the bite of an infected Aedes aegypti species mosquito. But it Zika can also be passed through sex from an infected to his or her sex partners and it can be spread from a pregnant woman to her fetus. Zika continues to spreading geographically to areas where competent vectors are present. Although a decline in cases of Zika virus infection has been reported in some countries, or in some parts of countries, vigilance needs to remain high. In this work, we propose a mathematical model that uses diffusion-advection equations to study the impact of the Zika epidemic. We present a numerical scheme linking finite elements (FEM) with finite differences to solve the model. The computer simulations are performed for Paramaribo and Santa Ana that have different demographic characteristics and allow us to extend the study to other regions.
\end{abstract}

Keywords. Diffusion, epidemic, model, Zika

\section{Resumen}

El virus del Zika se propaga a las personas principalmente a través de la picadura de un mosquito de la especie Aedes Aegypti infectado. El Zika también puede transmitirse a través del sexo de una persona infectada a sus parejas sexuales y se puede transmitir de una mujer embarazada a su feto. El Zika continúa expandiéndose geográficamente a áreas donde están presentes vectores competentes. Si bien se ha informado una disminución en los casos de infección por el virus del Zika en algunos países o en algunas partes de los países, la vigilancia debe mantenerse alta. En este trabajo proponemos un modelo matemático que utiliza ecuaciones de difusión-advección para estudiar el impacto de la epidemia de Zika. Presentamos un esquema numérico vinculando elementos finitos (FEM) con diferencias finitas para resolver el modelo. Las simulaciones computacionales se realizan para Paramaribo y Santa Ana, que tienen diferentes características demográficas y nos permiten ampliar el estudio a otras regiones.

Palabras clave. Difusión, epidemia, modelo, Zika.

1. Introduction. Zika virus is a mosquito-borne flavivirus that was first identified in Uganda in 1947 in monkeys through a network that monitored yellow fever. It was later identified in humans in 1952 in Uganda and the United Republic of Tanzania. Outbreaks of Zika virus disease have been recorded in Africa, the Americas, Asia and the Pacific. From the 1960s to 1980s, human infections were found across Africa and Asia, typically accompanied by mild illness. The first large outbreak of disease caused by Zika infection was reported from the Island

*IME- University of São Paulo, Rua do Matão, 1010, SP-Brazil (erickmath@ime. usp. br).

${ }^{\dagger}$ MATCOM- University of Havana, Edificio Felipe Poey. San Lázaro y L. Colina Universitaria, Havana-Cuba (aymee@mat com . uh . cu), 
of Yap (Federated States of Micronesia) in 2007 [5].

Zika virus is primarily transmitted to people through the bite of an infected mosquito from the Aedes genus, mainly Aedes aegypti in tropical regions. Aedes mosquitoes usually bite during the day, peaking during early morning and late afternoon/evening. This is the same mosquito that transmits dengue, chikungunya and yellow fever. Sexual transmission of Zika virus is also possible [4].

Recovery from Zika virus disease may require anywhere from 3 to 14 days after becoming infectious, but once recovered humans are believed to be immune from the virus for life, many people infected with Zika may be asymptomatic or will only display mild symptoms that do not require medical attention [4].

Sexual transmission of Zika virus is much more likely from men to women than from women to men, and same-sex transmission, from man to man, has only been documented once [13].

The use of diffusion and advection-diffusion equations in the study of epidemics can be seen in [10, 14], in particular for Dengue [9, 12, 17], for HIV / AIDS in [8, 15] and for Malaria in [11], these texts contributed background in the work that we present.

The objective of this work is to present a model for the Zika epidemic based in the diffusion-advection equations. To solve this model we use a numerical scheme that links elements and differences finites. Computational simulations are carried out in Paramaribo and Santa Ana, which are countries where Zika can develop endemically.

The paper is organized as follows. Section 1 is devoted to a Zika model by described by advection-diffusion equation. Section 2 presents the numerical method for the solution of the model. Section 3 is devoted to computer simulations for Santa Ana and Paramaribo. Section 4 are the conclusions of paper.

2. Construction of the Model. The model variables are susceptible men $H_{s}$, susceptible women $M_{s}$, infected men $H_{I}$, infected women $M_{I}$, recovered men $H_{R}$, recovered women $M_{R}$, susceptible mosquitoes $V_{s}$ and infected mosquitoes $V_{I}$. The parameters of the model are the force of infection from infected mosquito to susceptible human $\beta_{y_{1}}=$ (number of times a single mosquito bites a human per unit time $\times$ probability of pathogen transmission from an infectious mosquito to a susceptible human given that a contact between the two occurs) / the total population of human within the model). To define $\beta_{y_{2}}$ and $\beta_{y_{3}}$ we did an analogous study but taking into account the sexual contacts (between men and heterosexual respectively) and the probability of infecting these contacts, the force of infection from infected man to susceptible man by sexual contact $\beta_{y_{2}}$, the force of infection from infected man to susceptible woman by sexual contact $\beta_{y_{3}}$. The $\epsilon$ is a parameter modification associated with contagion by sexual contact with infected women. The force of infection from infected human to susceptible mosquito $\beta_{x}=$ (number of times a single mosquito bites a human per unit time $\times$ probability of pathogen transmission from an infectious human to a susceptible mosquito given that a contact between the two occurs) / the total population of human within the model, disease-induced death rate for man and woman $\epsilon_{1}, \epsilon_{2}$ and per capital recovery rate for humans from the infectious for man and woman $r_{1}, r_{2}$.

Let $l_{1}, l_{2}, l_{3}$ the life expectancy of men, women and mosquitoes. We define $\mu_{1}=\frac{1}{l_{1}}, \mu_{2}=\frac{1}{l_{2}}$ and $\eta=\frac{1}{l_{3}}$ such as death rates for men, women and mosquitoes respectively.

We define $N, M, V$ as the recruitment rate of men, women and mosquitoes (depends on the life expectancy and the total populations).

All model parameters are assumed to be positive. Description of model parameters are given in Table 2.1.

Assumptions for the construction of the model:

- There is immunity in the recovered state, the infected man can infect a susceptible woman and a susceptible man (result of the study of other epidemics that are transmitted by sexual contact).

- The contagion to the fetus is not taken into account, because the fetus is not directly in the transmission dynamics.

- The death by natural causes is equal in any state, the death of mosquitoes will be due to environmental factors because no control strategy is applied.

- We do not take into account the contagion among women in any of the compartments.

- By definition epidemiological $H_{s}, M_{s}, H_{I}, M_{I}, H_{R}, M_{R}, V_{s}$ and $V_{I}$ and the initial conditions are continuous functions and positive or null.

The model represents the transmission with exposed variable, it is a SIR model (susceptible-infected-recovered) for humans and for mosquitoes we do not have the status of recovered.

The Laplacian $(\Delta)$ and the gradient $(\nabla)$ operators are relative to the spatial variable $x \in \Omega$ and assume that the 


\begin{tabular}{|l|l|}
\hline Parameters & Description \\
\hline$\beta_{y_{1}}$ & The force of infection from infected mosquito to susceptible human \\
\hline$\beta_{y_{2}}$ & The force of infection from infected man to susceptible man \\
\hline$\beta_{y_{3}}$ & The force of infection from infected man to susceptible woman \\
\hline$\beta_{x}$ & The force of infection from infected human to susceptible mosquito \\
\hline$\mu_{1}, \mu_{2}, \eta$ & Man, woman and mosquito mortality rate \\
\hline$\epsilon_{1}, \epsilon_{2}$ & Disease-induced death rate for humans (men and women) \\
\hline$r_{1}, r_{2}$ & Per capital recovery rate for humans from the infectious (men and women) \\
\hline$N, M, V$ & Recruitment rate of men, women and mosquitoes \\
\hline
\end{tabular}

Table 2.1: Description of parameters used in the model (1).

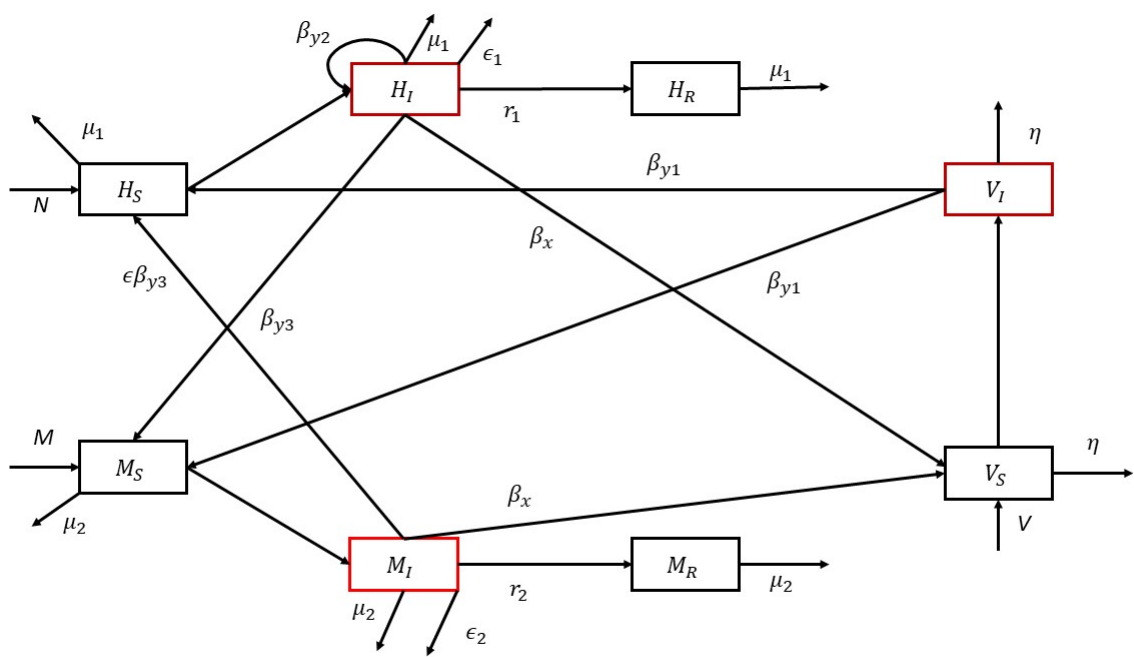

Figure 2.1: This schematic representation shows the Zika progression in human and mosquito populations for our model. Susceptible humans start in $H_{s}, M_{s}$ (men and women) and move to $H_{I}$ and $M_{I}$, the infected population, once infected by a mosquito carrying the virus and sexual contact. Infectious humans will then move to and remain in $H_{R}$ and $M_{R}$ after recovering from the infection. The loop in the compartment of infected men with $\beta_{y_{2}}$ represents co-infection. The susceptible mosquitoes population is denoted $V_{s}$. After transmission occurs from biting an infectious human, susceptible mosquitoes transition to the infected population, $V_{I}$, where they remain infectious until death.

initial data are continuous and bounded functions on $\bar{\Omega}$.

$$
\begin{aligned}
& \frac{\partial H_{s}}{\partial t}-\nabla \cdot\left(\alpha_{s} \nabla H_{s}\right)+\nabla \cdot\left(\beta_{s} H_{s}\right)=N-\beta_{y_{1}} H_{s} V_{I}-\beta_{y_{2}}\left(H_{I}+\epsilon M_{I}\right) H_{s}-\mu_{1} H_{s}, \\
& \frac{\partial M_{s}}{\partial t}-\nabla \cdot\left(\alpha_{s} \nabla M_{s}\right)+\nabla \cdot\left(\beta_{s} M_{s}\right)=M-\beta_{y_{1}} M_{s} V_{I}-\beta_{y_{3}} M_{s} H_{I}-\mu_{2} M_{s}, \\
& \frac{\partial H_{I}}{\partial t}-\nabla \cdot\left(\alpha_{I} \nabla H_{I}\right)+\nabla \cdot\left(\beta_{I} H_{I}\right)=\beta_{y_{1}} H_{s} V_{I}+\beta_{y_{2}}\left(H_{I}+\epsilon M_{I}\right)-\left(r_{1}+\epsilon_{1}+\mu_{1}\right) H_{I}, \\
& \frac{\partial M_{I}}{\partial t}-\nabla \cdot\left(\alpha_{I} \nabla M_{I}\right)+\nabla \cdot\left(\beta_{I} M_{I}\right)=\beta_{y_{1}} M_{s} V_{I}+\beta_{y_{3}} M_{s} H_{I}-\left(r_{2}+\epsilon_{2}+\mu_{2}\right) M_{I}, \\
& \frac{\partial H_{R}}{\partial t}-\nabla \cdot\left(\alpha_{r} \nabla H_{R}\right)+\nabla \cdot\left(\beta_{r} H_{R}\right)=r_{1} H_{I}-\mu_{1} H_{R}, \\
& \frac{\partial M_{R}}{\partial t}-\nabla \cdot\left(\alpha_{r} \nabla M_{R}\right)+\nabla \cdot\left(\beta_{r} M_{R}\right)=r_{2} M_{I}-\mu_{2} M_{R}, \\
& \frac{\partial V_{s}}{\partial t}-\nabla \cdot\left(\alpha_{m} \nabla V_{s}\right)+\nabla \cdot\left(\beta_{m} V_{s}\right)=V-\beta_{x} V_{s}\left(H_{I}+M_{I}\right)-\eta V_{s}, \\
& \frac{\partial V_{I}}{\partial t}-\nabla \cdot\left(\alpha_{p} \nabla V_{I}\right)+\nabla \cdot\left(\beta_{p} V_{I}\right)=\beta_{x} V_{s}\left(H_{I}+M_{I}\right)-\eta V_{I} .
\end{aligned}
$$

Boundary condition (zero influx conditions):

$$
\frac{\partial H_{s}\left(t, x^{*}\right)}{\partial \xi}=\frac{\partial H_{I}\left(t, x^{*}\right)}{\partial \xi}=\frac{\partial H_{R}\left(t, x^{*}\right)}{\partial \xi}=\frac{\partial M_{s}\left(t, x^{*}\right)}{\partial \xi}=\frac{\partial M_{I}\left(t, x^{*}\right)}{\partial \xi}=\frac{\partial M_{R}\left(t, x^{*}\right)}{\partial \xi}=\frac{\partial V_{s}\left(t, x^{*}\right)}{\partial \xi}=\frac{\partial V_{I}\left(t, x^{*}\right)}{\partial \xi}=
$$

The homogeneous Neumann boundary conditions mean that there is no population flux across the boundary $\partial \Omega$ and both the human and mosquito individuals live in a self-contained environment. The $\xi$ is the outward normal 
vector to $\partial \Omega$.

The $\alpha_{s}, \alpha_{I}$ and $\alpha_{r}$ are the dispersion rate for susceptible, infected and recovered humans and $\beta_{s}, \beta_{I}$ and $\beta_{r}$ are the velocities field relative to the migratory movement of susceptible, infected and recovered humans, respectively. We will consider the mosquito dispersal as the result of a random (and local) flying movement, macroscopically represented by a diffusion process with coefficients $\alpha_{m}$ and $\alpha_{p}$, coupled to a wind advection caused by a constants velocity flux $\beta_{m}$ and $\beta_{p}$. Constant advection can be justified as a bias in the transport process caused by a longterm geographical direction of the wind, while its random and short-term fluctuations are to be included in the diffusion term.

In [7], mathematical models similar to the right member of model (1) were presented, where the transmission of Zika is modelled but with exposed variables and time delay. A mathematical and epidemiological study of the models and computational simulations for Suriname and El Salvador was carried out. This study evidenced the influence of parameters $\beta_{y_{1}}$ and $\beta_{x}$ (related with contagion by mosquito bites) in the transmission of Zika and that sexual contagion does not exert a strong influence.

3. Method of solution. First we find the variational formulation of the model and apply the Galerkin method, [2], [3]

Let $W=\left\{L^{2}\left(\left[0, T_{f}\right], V\right)\right\}, V=H^{1}(W)$ space of test functions and we define the scalar product:

$$
\langle u, v\rangle=\int_{\Omega} u v d x d y, \quad\langle\nabla u|| \nabla v\rangle=\int_{\Omega} \nabla u \nabla v d \mu
$$

$u \in W, v \in V$.

Let $U=H_{s}, M_{s}, H_{I}, M_{I}, H_{R}, M_{R}, V_{s}, V_{I}, U(t, x, y)=U$,

$v=v(x, y),(x, y) \in \Omega$, by the formula of Green

$$
-\alpha_{U}\langle\Delta U, v\rangle=\alpha_{U}\langle\nabla U \| \nabla v\rangle-\alpha_{U}\left\langle\frac{\partial U}{\partial \eta}, v\right\rangle
$$

Remembering that we have boundary condition null.

We write the parameters (constants in this study) as follows:

$$
\begin{aligned}
& \beta_{s}=\left\langle\beta_{s_{1}}, \beta_{s_{2}}\right\rangle, \\
& \beta_{I}=\left\langle\beta_{I_{1}}, \beta_{I_{2}}\right\rangle, \\
& \beta_{r}=\left\langle\beta_{r_{1}}, \beta_{r_{2}}\right\rangle, \\
& \beta_{m}=\left\langle\beta_{m_{1}}, \beta_{m_{2}}\right\rangle, \\
& \beta_{p}=\left\langle\beta_{p_{1}}, \beta_{p_{2}}\right\rangle .
\end{aligned}
$$

Let $\left\{\phi_{i}\right\}_{i=1}^{n}$ base of $V_{h} \subset V$ be a finite approximation of the problem. We use the notation:

$$
U_{h}=\sum_{j=1}^{n} U_{j}(t) \phi_{j}(x, y)
$$

The respective derivatives are:

$$
\frac{\partial U_{h}}{\partial t}=\sum_{j=1}^{n} \frac{d U_{j}}{d t} \phi_{j}(x, y), \frac{\partial U_{h}}{\partial x}=\sum_{j=1}^{n} U_{j} \frac{\partial \phi_{j}}{\partial x}, \frac{\partial U_{h}}{\partial y}=\sum_{j=1}^{n} U_{j} \frac{\partial \phi_{j}}{\partial y}
$$

In this approximation, the model is expressed as: 


$$
\begin{aligned}
& \left\langle\frac{\partial H_{s}}{\partial t}, v\right\rangle+\alpha_{s}\left\langle\nabla H_{s}|| \nabla v\right\rangle+\beta_{s_{1}}\left\langle\frac{\partial H_{s}}{\partial x}, v\right\rangle+\beta_{s_{2}}\left\langle\frac{\partial H_{s}}{\partial y}, v\right\rangle=N-\beta_{y_{1}}\left\langle H_{s} V_{I}, v\right\rangle \\
& -\beta_{y_{2}}\left\langle H_{s} H_{I}, v\right\rangle-\mu_{1}\left\langle H_{s}, v\right\rangle, \\
& \left\langle\frac{\partial M_{s}}{\partial t}, v\right\rangle+\alpha_{s}\left\langle\nabla M_{s}|| \nabla v\right\rangle+\beta_{s_{1}}\left\langle\frac{\partial M_{s}}{\partial x}, v\right\rangle+\beta_{s_{2}}\left\langle\frac{\partial M_{s}}{\partial y}, v\right\rangle=M-\beta_{y_{1}}\left\langle M_{s} V_{I}, v\right\rangle \\
& -\beta_{y_{3}}\left\langle M_{s} H_{I}, v\right\rangle-\mu_{2}\left\langle M_{s}, v\right\rangle, \\
& \left\langle\frac{\partial H_{I}}{\partial t}, v\right\rangle+\alpha_{I}\left\langle\nabla H_{I}|| \nabla v\right\rangle+\beta_{I_{1}}\left\langle\frac{\partial H_{I}}{\partial x}, v\right\rangle+\beta_{I_{2}}\left\langle\frac{\partial H_{I}}{\partial y}, v\right\rangle=\beta_{y_{1}}\left\langle H_{s} V_{I}, v\right\rangle \\
& +\beta_{y_{2}}\left\langle H_{s} H_{I}, v\right\rangle-\left(r_{1}+\epsilon_{1}+\mu_{1}\right)\left\langle H_{I}, v\right\rangle, \\
& \left\langle\frac{\partial M_{I}}{\partial t}, v\right\rangle+\alpha_{I}\left\langle\nabla M_{I}|| \nabla v\right\rangle+\beta_{I_{1}}\left\langle\frac{\partial M_{I}}{\partial x}, v\right\rangle+\beta_{I_{2}}\left\langle\frac{\partial M_{I}}{\partial y}, v\right\rangle=\beta_{y_{1}}\left\langle M_{s} V_{I}, v\right\rangle \\
& +\beta_{y_{2}}\left\langle M_{s} H_{I}, v\right\rangle-\left(r_{2}+\epsilon_{2}+\mu_{2}\right)\left\langle M_{I}, v\right\rangle, \\
& \left\langle\frac{\partial H_{R}}{\partial t}, v\right\rangle+\alpha_{r}\left\langle\nabla H_{R} \| \nabla v\right\rangle+\beta_{r_{1}}\left\langle\frac{\partial H_{R}}{\partial x}, v\right\rangle+\beta_{r_{2}}\left\langle\frac{\partial H_{R}}{\partial y}, v\right\rangle=r_{1}\left\langle H_{I}, v\right\rangle-\mu_{1}\left\langle H_{R}, v\right\rangle, \\
& \left\langle\frac{\partial M_{R}}{\partial t}, v\right\rangle+\alpha_{r}\left\langle\nabla M_{R} \| \nabla v\right\rangle+\beta_{r_{1}}\left\langle\frac{\partial M_{R}}{\partial x}, v\right\rangle+\beta_{r_{2}}\left\langle\frac{\partial M_{R}}{\partial y}, v\right\rangle=r_{2}\left\langle M_{I}, v\right\rangle-\mu_{2}\left\langle M_{R}, v\right\rangle, \\
& \left\langle\frac{\partial V_{s}}{\partial t}, v\right\rangle+\alpha_{m}\left\langle\nabla V_{s} \| \nabla v\right\rangle+\beta_{m_{1}}\left\langle\frac{\partial V_{s}}{\partial x}, v\right\rangle+\beta_{m_{2}}\left\langle\frac{\partial V_{s}}{\partial y}, v\right\rangle=V-\beta_{x}\left\langle V_{s}\left(H_{I}+M_{I}\right), v\right\rangle \\
& -\eta\left\langle V_{s}, v\right\rangle, \\
& \left\langle\frac{\partial V_{I}}{\partial t}, v\right\rangle+\alpha_{m}\left\langle\nabla V_{I}|| \nabla v\right\rangle+\beta_{m_{1}}\left\langle\frac{\partial V_{I}}{\partial x}, v\right\rangle+\beta_{m_{2}}\left\langle\frac{\partial V_{I}}{\partial y}, v\right\rangle=\beta_{x}\left\langle V_{s}\left(H_{I}+M_{I}\right), v\right\rangle \\
& -\eta\left\langle V_{I}, v\right\rangle,
\end{aligned}
$$

For the temporary variables we use the Crank-Nicolson method (central differences in the time $\left.t_{n+\frac{1}{2}}\right)$ was used

$$
\begin{aligned}
\frac{U_{j}}{d t}\left(x, y, t_{n+\frac{1}{2}}\right) & =\frac{U_{j}^{n+1}-U_{j}^{n}}{2} . \\
\frac{d U_{j}}{d t}\left(x, y, t_{n+\frac{1}{2}}\right) & =\frac{U_{j}^{n+1}-U_{j}^{n}}{\Delta t}
\end{aligned}
$$

The matrix formulation is:

$$
\begin{aligned}
& C_{h s}\left(H_{s}^{n}, H_{s}^{n+1}, V_{I}^{n+1}, V_{I}^{n}, H_{I}^{n+1}, H_{I}^{n}\right) H_{s}^{n+1}=D_{h s}\left(H_{s}^{n},\right. \\
& \left.H_{s}^{n+1}, V_{I}^{n+1}, V_{I}^{n}, H_{I}^{n+1}, H_{I}^{n}\right) H_{s}^{n}+E_{1}, \\
& C_{m s}\left(M_{s}^{n}, M_{s}^{n+1}, V_{I}^{n+1}, V_{I}^{n}, H_{I}^{n+1}, H_{I}^{n}\right) M_{s}^{n+1}=D_{m s}\left(M_{s}^{n},\right. \\
& \left.M_{s}^{n+1}, V_{I}^{n+1}, V_{I}^{n}, H_{I}^{n+1}, H_{I}^{n}\right) M_{s}^{n}+E_{2}, \\
& C_{h I}(c) H_{I}^{n+1}=D_{I}\left(H_{s}^{n}, H_{s}^{n+1}, V_{I}^{n+1}, V_{I}^{n}, H_{I}^{n+1}, H_{I}^{n}\right) H_{I}^{n}+E_{3}, \\
& C_{m I}(c) M_{I}^{n+1}=D_{I}\left(M_{s}^{n}, M_{s}^{n+1}, V_{I}^{n+1}, V_{I}^{n}, H_{I}^{n+1}, H_{I}^{n}\right) M_{I}^{n}+E_{4}, \\
& C_{h R}(c) H_{R}^{n+1}=D_{R}\left(H_{I}^{n+1}, H_{I}^{n}, H_{R}^{n+1}, H_{R}^{n}\right) H_{R}^{n}+E_{5}, \\
& C_{m R}(c) H_{R}^{n+1}=D_{R}\left(M_{I}^{n+1}, M_{I}^{n}, M_{R}^{n+1}, M_{R}^{n}\right) M_{R}^{n}+E_{6}, \\
& C_{v S}\left(V_{s}^{n+1}, V_{s}^{n}, H_{I}^{n+1}, H_{I}^{n}, M_{I}^{n+1}, M_{I}^{n}\right) V_{s}^{n+1}=D_{v S}\left(V_{s}^{n+1},\right. \\
& \left.V_{s}^{n+1}, H_{I}^{n+1}, H_{I}^{n}, M_{I}^{n+1}, M_{I}^{n}\right) V_{s}^{n}+E_{7}, \\
& C_{v I}(c) V_{I}^{n+1}=D_{v I}\left(H_{I}^{n+1}, H_{I}^{n}, M_{I}^{n+1}, M_{I}^{n}\right) V_{I}^{n}+E_{8} .
\end{aligned}
$$

Where $C_{h I}(c), C_{m I}(c), C_{h R}(c), C_{m R}(c)$ and $C_{v I}(c)$ are matrices of constant coefficients.

The $E_{i} i=1,2, \ldots, 8$ depend on some of the variables and parameters of the model (non-linearity of system).

Solution algorithm proposal for (2):

To solve the system we used a predictor-corrector method [1, 2].

Using $H_{s}^{0}, M_{s}^{0}, H_{I}^{0}, M_{I}^{0}, H_{R}^{0}, M_{R}^{0}, V_{s}^{0}$, and $V_{I}^{0}$,

we calculate $H_{s}^{1}, M_{s}^{1}, H_{I}^{1}, M_{I}^{1}, H_{R}^{1}, M_{R}^{1}, V_{s}^{1}$, and $V_{I}^{1}$.

- We calculate $H_{s}^{*}, M_{s}^{*}$ in:

$C_{h_{s}}\left(H_{s}^{0}, H_{s}^{0}, V_{I}^{0}, V_{I}^{0}, H_{I}^{0}, H_{I}^{0}\right) H_{s}^{*}=D_{h s}\left(H_{s}^{0}, H_{s}^{0}, V_{I}^{0}, V_{I}^{0}, H_{I}^{0}, H_{I}^{0}\right) H_{s}^{0}+E_{1}^{0}$

$C_{m s}\left(M_{s}^{0}, M_{s}^{0}, V_{I}^{0}, V_{I}^{0}, H_{I}^{0}, H_{I}^{0}\right) M_{s}^{*}=D_{m s}\left(M_{s}^{0}, M_{s}^{0}, V_{I}^{0}, V_{I}^{0}, H_{I}^{0}, H_{I}^{0}\right) M_{s}^{0}+E_{2}^{0}$.

The $E_{1}^{0}$ and $E_{2}^{0}$ depend of the initial conditions and from this iteration the $E_{i} i=1,2, \ldots, 8$ are updated.

- We calculate $H_{I}^{*}, M_{I}^{*}$ in:

$C_{h I}(c) H_{I}^{*}=D_{I}\left(H_{s}^{0}, H_{s}^{*}, V_{I}^{0}, V_{I}^{0}, H_{I}^{0}, H_{I}^{0}\right) H_{I}^{0}+E_{3}$,

$C_{m I}(c) I^{*}=D_{I}\left(M_{s}^{0}, M_{s}^{*}, V_{I}^{0}, V_{I}^{0}, H_{I}^{0}, H_{I}^{0}\right) M_{I}^{0}+E_{4}$, 
- We calculate $H_{R}^{*}, M_{R}^{*}$ in:

$C_{h R}(c) R^{*}=D_{R}\left(H_{I}^{*}, H_{I}^{0}, H_{R}^{0}, H_{R}^{0}\right) H_{R}^{0}+E_{5}$,

$C_{m R}(c) R^{*}=D_{R}\left(M_{I}^{*}, M_{I}^{0},, M_{R}^{0}, M_{R}^{0}\right) M_{R}^{0}+E_{6}$,

- We calculate $V_{s}^{*}, V_{I}^{*}$ in:

$C_{v S}\left(V_{s}^{0}, V_{s}^{0}, H_{I}^{0}, H_{I}^{*}, M_{I}^{0}, M_{I}^{*}\right) V_{s}^{*}=D_{v S}\left(V_{s}^{0}, V_{s}^{*}, H_{I}^{0}, H_{I}^{*}, M_{I}^{0}, M_{I}^{*}\right) V_{s}^{0}+E_{7}$

$C_{v I}(c) V_{I}^{*}=D_{v I}\left(H_{I}^{0}, H_{I}^{*}, M_{I}^{0}, M_{I}^{*}\right) V_{I}^{0}+E_{8}$

- We calculate $H_{s}^{* *}, M_{s}^{* *}$ in:

$C_{h s}\left(H_{s}^{0}, H_{s}^{*}, V_{I}^{0}, V_{I}^{*}, H_{I}^{0}, H_{I}^{*}\right) H_{s}^{* *}=D_{h s}\left(H_{s}^{0}, H_{s}^{*}, V_{I}^{0}, V_{I}^{*}, H_{I}^{0}, H_{I}^{*}\right) H_{s}^{0}+E_{1}$,

$C_{m s}\left(M_{s}^{0}, M_{s}^{*}, V_{I}^{0}, V_{I}^{*}, H_{I}^{0}, H_{I}^{*}\right) M_{s}^{* *}=D_{m s}\left(M_{s}^{0}, M_{s}^{*}, V_{I}^{0}, V_{I}^{*}, H_{I}^{0}, H_{I}^{*}\right) M_{s}^{0}+E_{2}$,

- We calculate $H_{I}^{* *}, M_{I}^{* *}$ in:

$C_{h I}(c) H_{I}^{* *}=D_{I}\left(H_{s}^{0}, H_{s}^{* *}, V_{I}^{0}, V_{I}^{*}, H_{I}^{0}, H_{I}^{* *}\right) H_{I}^{0}+E_{3}$,

$C_{m I}(c) M_{I}^{* *}=D_{I}\left(M_{s}^{0}, M_{s}^{* *}, V_{I}^{0}, V_{I}^{*}, H_{I}^{0}, H_{I}^{*}\right) M_{I}^{0}+E_{4}$,

- We calculate $H_{R}^{* *}, M_{R}^{* *}$ in:

$C_{h R}(c) H_{R}^{* *}=D_{R}\left(H_{I}^{* *}, H_{I}^{0}, H_{R}^{0}, H_{R}^{*}\right) H_{R}^{0}+E_{5}$,

$C_{m R}(c) M_{R}^{* *}=D_{R}\left(M_{I}^{* *}, M_{I}^{0}, M_{R}^{0}, M_{R}^{*}\right) M_{R}^{0}+E_{6}$,

- We calculate $V_{s}^{* *}, V_{I}^{* *}$ in:

$C_{v S}\left(V_{s}^{0}, V_{s}^{*}, H_{I}^{0}, H_{I}^{* *}, M_{I}^{0}, M_{I}^{* *}\right) V_{s}^{* *}=D_{v S}\left(V_{s}^{0}, V_{s}^{*}, H_{I}^{0}, H_{I}^{* *}, M_{I}^{0}, M_{I}^{* *}\right) V_{s}^{0}$

$+E_{7}$,

$C_{v I}(c) V_{I}^{* *}=D_{v I}\left(H_{I}^{0}, H_{I}^{* *}, M_{I}^{0}, M_{I}^{* *}\right) V_{I}^{0}+E_{8}$

For k iterations, with certain condition of stop of the process you have:

$H_{s}^{1}=H_{s}^{k *}, M_{s}^{1}=M_{s}^{k *}, H_{I}^{1}=H_{I}^{k *}, M_{I}^{1}=M_{I}^{k *}, H_{R}^{1}=H_{R}^{k *}, M_{R}^{1}=M_{R}^{k *}, V_{s}^{1}=V_{s}^{k *}, V_{I}^{1}=V_{I}^{k *}$,

and we continue the process until we obtain the approximate solution of the model.

4. Discussion. The computational experimentation are carried out for Paramaribo and Santa Ana, which have different demographic and climatic characteristics and Zika can become an endemic problem. The values of parameters and initial conditions were extracted from [12, 16, 17] and some were assumed after discussing with specialists in this epidemic (to use logical values not far from reality). The Matlab-R2017a software was used for programming. The $\Omega$ was taken as the unit circle to obtain adequate graphic results but for future works it will be carried out in the regions under study directly. Was chosen a season in which it is prone to the development of the mosquito. All sub-populations were studied but by relevance we present the results for mosquitoes and infected men.

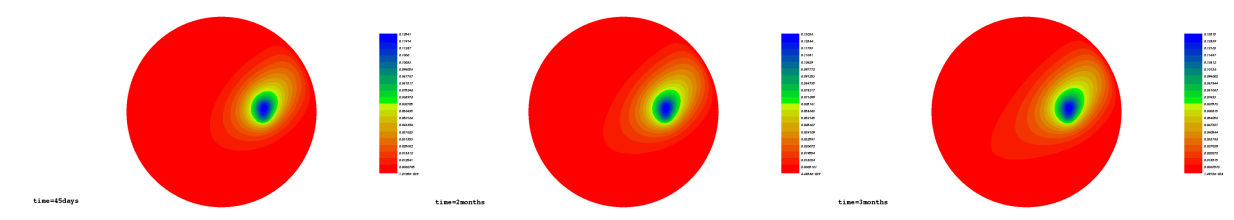

Figure 4.1: Behavior of infected men in Paramaribo at 45 days, 2 months and 3 months (Result of the investigation).

The diffusion of infected men in Paramaribo is greater compared to Santa Ana and the behavior is to the border of the region in both scenarios, see figures (4.1) and (4.2).

Among other results we have that infected men spread with greater speed and space than infected women, but both move to the border of the region and a greater number of recovered men than women is reported over time, which shows that the greater the number of infected people, the greater the number recovered for this epidemic in both scenarios.

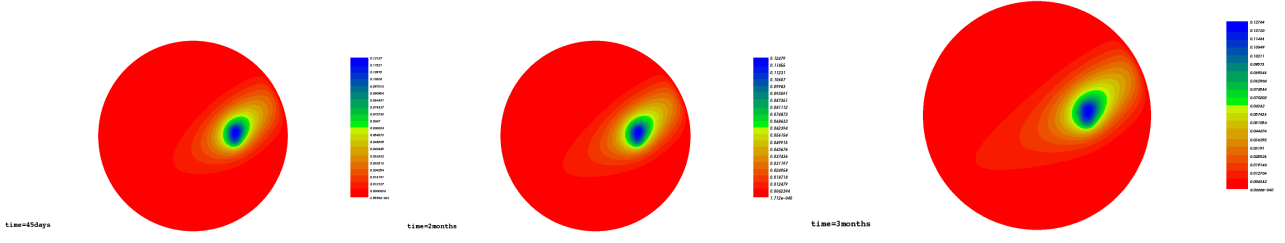

Figure 4.2: Behavior of infected men in Santa Ana at 45 days, 2 months and 3 months (Result of the investigation). 
Infected mosquitoes spread more strongly in Santa Ana than in Paramaribo, logical behavior as the population of humans in Santa Ana is larger. But in both scenarios the mosquitoes, the diffusion is to the interior of the region contrary to the humans who move to the border, see figures (4.3) and (4.4).

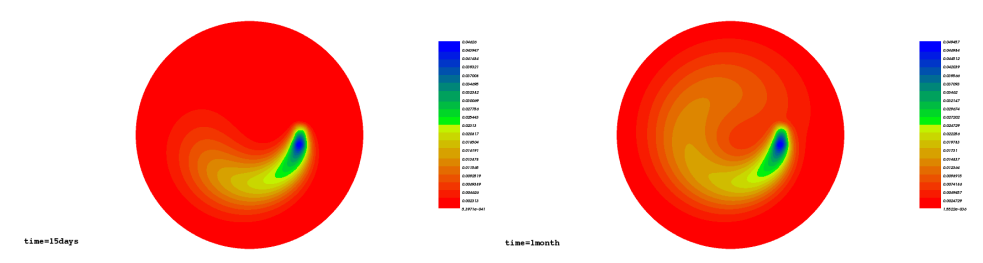

Figure 4.3: Behavior of infected mosquitoes in Paramaribo at 15 days and 1 month (Result of the investigation).

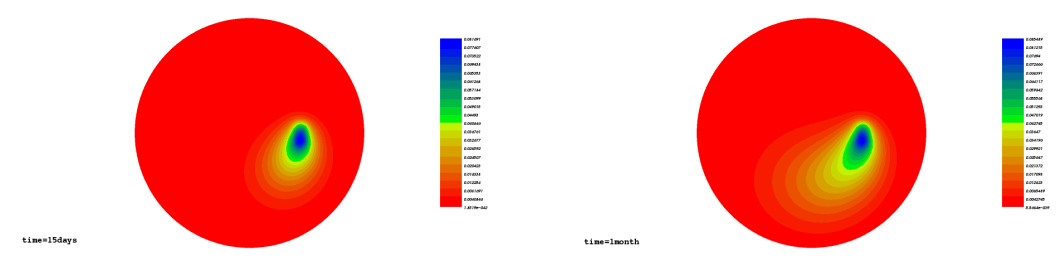

Figure 4.4: Behavior of infected mosquitoes in Santa Ana at 15 days and 1 month (Result of the investigation).

5. Conclusions. We present a mathematical model of the epidemic of Zika that allows to investigate the spread of the disease over time. Among the results of the computational experimentation is that the spread of infected humans in Paramaribo is greater than in Santa Ana and they move towards the border of the region. In the case of infected mosquitoes, Santa Ana shows the greatest diffusion compared to Paramaribo and, in general, infected mosquitoes spread more rapidly than infected humans and move into the interior of the region. These results show the need to apply an adequate control strategy because Zika can become endemic in both scenarios. For future work we will use the involution operator for the diffusion of humans because it is closer to behavior in reality and will take into account the time delay for humans and mosquitoes that will be the time it takes to develop the pathogen.

6. Acknowledgements. We thank CAPES for the financial support and the Institute of Mathematics and Statistics of the University of São Paulo.

ORCID and License

Erick Manuel Delgado Moya https://orcid.org/0000-0001-5937-5374, Aymee Marrero Severo https://orcid.org/0000-0003-4823-0078.

This work is licensed under the Creative Commons Attribution-NoComercial-ShareAlike 4.0. 
References

[1] Butcher, J. C. Numerical method for ordinary differential equations, John Wiley and Sons, 2003. DOI: $10.1002 / 9780470753767$.

[2] Ciarlet, P. G. The finite element method for elliptic problem, SIAM, 2002. ISBN: 978-0898715149.

[3] Crank, J. and Nicolson, E. A practical method for numerical evaluation of partial differential equations of the heat conduction type, Proc. Camb. Phil. Soc., 1947; 43(1):50-67. DOI: 10.1017/S0305004100023197.

[4] Dick, G. W. A., Kitchen, S. F. and Haddow, A. J. Zika Virus. I. Isolations and serogical specifity, Trans R Soc Trop Med Hyg, 1952; 46(5):509-520. DOI: 10.1016/0035-9203(52)90042-4.

[5] Dick, G. W. A., Kitchen, S. F. and Haddow, A. J. Zika virus (II). Pathogenicity and physical properties, Trans R Soc Trop Med Hyg, 1952; 46:521-534. DOI: 10.1016/0035-9203(52)90043-6.

[6] Bonyah Ebenezer and Okosun Kazeem. Mathematical Modelling of Zika virus. Asian Pacific Journal of Tropical Disease. doi: 10.1016/S2222-1808(16)61108-8

[7] Delgado, E. M. and Marrero S. A. Mathematical models for Zika with exposed variables and delay. Comparison and experimentation in Suriname and El Salvador, Selecciones Matemáticas, ISSN: 2411-1783, 2019. DOI: 10.17268/sel.mat.2019.01.01.

[8] Kassen, T. G. and Garba, E. J. D. A mathematical model for the spatial spread oh HIV in heterogeneous population, Mathematical Theory and Modeling, 2016; 6(4):95-104. ISSN: 2224-5804.

[9] Lin, H. and Wang, F. On a reaction-diffusion system modeling the Dengue transmission with nonlocal infections and crowding effects, Applied Mathematics and Computation, 2014; 248:184-194. DOI: 10.1016/j.amc.2014.09.101.

[10] Lotfi, E. M., Maziane, M., Hattaf, K. and Yousti, N. Partial differential equations of an epidemic model with spatial difussion, International Journal of Partial Differential Equations, volume 2014, article ID 186437, 2014. DOI: 10.1155/2014/186437

[11] Macufa, M. M., Mayer, J. F. and Krindges, A. Difussion of Malaria in Mozambique. Modeling with computational simulations, Biomatemática, 2015; 25:161-184.

[12] Maidana, N. A. and Yang, H. M. A spatial model to describe the Dengue propagation, TEMA, Tend. Mat. Apli. Comp., 2007; 8(1):83-92. ISSN: 1677-1966.

[13] Prevention of sexual transmission of Zika virus: interim guidance update, Geneva: World Health Organization; 2016 (WHO/ZIKV/MOC/16.1 Rev.3; https://apps.who.int/iris/handle/10665/204421)

[14] Peng, R. and Zhao, X. Q. A reaction-diffusion SIS epidemic model in a time periodic environment, Nonlinearity, $2012 ; 25: 1451-1471$. DOI: $10.1088 / 0951-7715 / 25 / 5 / 1451$.

[15] X. Ren, Tian, Y., Liu, L. and Liu, X. A reaction difussion withen-host HIV model with cell-to-cell transmision, J. Math. Biol., 2018; 76:1831-1872. DOI: 10.1007/s00285-017-1202-X.

[16] Shutt, D. P., Manore, C. A., Pankavich, S., Porter, A. T. and del Valle, S. Y. Estimating the reproductive number, total outbreak size, and reporting rates for ZIKA epidemics in South and Central America, Epidemics, 2017; 21:63-79. DOI: 10.1016/j.epidem.2017.06.005.

[17] Tiemi, T. T., Maidan, N. A., Ferreira Jr, W. C., Paulino, P. and Yang, H. Mathematical Models for the Aedes aegypti dispersal dynamics: Travelling waves by wing and wind, Bulletin of Mathematical Biology, 2005; 67:509-528. DOI: 10.1016/j.bulm.2004.08.005. 